Arthroskopie 2010 $23: 250-251$ DOI 10.1007/s00142-010-0576-1 Online publiziert: 21. Oktober 2010 (c) Springer-Verlag 2010

\author{
J.D. Agneskirchner ${ }^{1} \cdot$ W. Anderl ${ }^{2}$ \\ ${ }^{1}$ Sportsclinic Germany, Hannover \\ ${ }^{2}$ Orthopädische Abteilung, Krankenhaus der Barmherzigen Schwestern Wien
}

\title{
Erkrankungen des Schultergürtels
}

Liebe Leserinnen und Leser,

Die Arthroskopie des Schultergelenks hat sich in den letzten Jahren rasant entwickelt. Effektive Techniken zur arthroskopischen Therapie von Läsionen an Labrum, Ligamenten, Bizepssehne und Rotatorenmanschette stehen zur Verfügung und sind zunehmend etabliert. Im Fokus der diagnostischen und therapeutischen Maßnahmen liegen dabei meist intraartikuläre und subakromiale Pathologien.

Mit den Beiträgen in diesem Heft wollen wir den Blick auf die dem Glenohumeralgelenk benachbarten Gelenke und Strukturen richten. Die Anatomie des Schultergürtels gestattet wie bei keinem anderen Gelenk auch einen arthroskopischen Zugang zu den an das Glenohumeralgelenk angrenzenden extraartikulären Räumen und benachbarten Gelenken, wobei sich hier schon einige arthroskopische Behandlungstechniken entwickelt haben.

In Übersichtsartikeln werden die Pathologien und Behandlungsoptionen des Skapulothorakal- und Sternoklavikulargelenks, die meist recht wenig beachtet werden, beschrieben. Des Weiteren werden Operationstechnik und Ergebnisse bei der arthroskopischen AC-Gelenkresektion sowie aktuelle arthroskopische Stabilisierungstechniken bei akuter und chronischer AC-Gelenkinstabilität dargestellt.

Im Beitrag über die sekundären Impingementsyndrome der Schulter werden praktisch relevante Hinweise zum Verständnis der schwierigen Zusammenhänge zwischen pathologischen Bewegungs- mustern der Schulter und sekundären intraartikulären Läsionen gegeben.

Eine weitere Arbeit widmet sich der arthroskopischen Behandlung von Ganglien und Nervenentrapmentsyndromen des Schultergürtels, wobei sich hier für den Arthroskopeur die faszinierende Möglichkeit der direkten, arthroskopisch kontrollierten Ganglionresektion und Nervendekompression ergibt.

Ziel dieses Themenhefts ist es, Ihnen mit der Lektüre einen aktuellen Überblick über die Pathologien und Behandlungsoptionen der Strukturen des Schultergürtels zu geben und Sie darüber hinaus für neue arthroskopische Therapieansätze in diesem Bereich zu sensibilisieren, die noch weiterer kritischer Evaluation bedürfen. Ihre

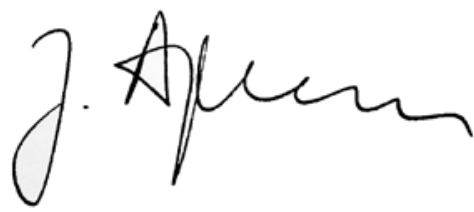

Dr. Jens D. Agneskirchner

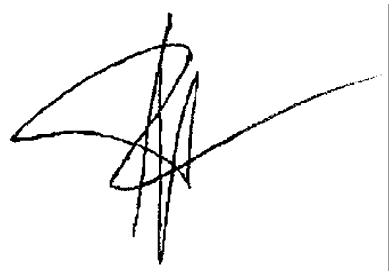

Dr. Werner Anderl

\section{Korrespondenzadresse \\ Dr. J.D. Agneskirchner}

Sportsclinic Germany

Uhlemeyerstraße 16, 30175 Hannover

jens.agneskirchner@sportsclinicgermany.com 


\section{Hier steht eine Anzeige.}

第 Springer 